Bull. Austral. Math. Soc.

$26 \mathrm{~A} 51,90 \mathrm{c} 30$

VOL. 65 (2002) [289-306]

\title{
INVEXITY OF SUPREMUM AND INFIMUM FUNCTIONS
}

\author{
NguYen Xuan Ha and Do Van LuU
}

\begin{abstract}
Under suitable assumptions we establish the formulas for calculating generalised gradients and generalised directional derivatives in the Clarke sense of the supremum and the infimum of an infinite family of Lipschitz functions. From these results we derive the results ensuring such a supremum or infimum are an invex function when all functions of the family are invex. Applying these results to a class of mathematical programs, we obtain necessary and sufficient conditions for optimality.
\end{abstract}

\section{INTRODUCTION}

The theory of invex functions has been extensively studied by many authors (see, for example, $[1,4,5,6,7,8,10,11,12,14,15])$. The works of Hanson [8] and Craven [10] are the starting points of this theory. This research was inspired by the recent work of Luu and $\mathrm{Ha}$ [12], who proved that the minimum and the maximum of a finite family of invex functions defined on a Banach space are invex functions under suitable hypotheses. To study the supremum and the infimum of an infinite family of invex Lipschitz functions, we need to calculate their Clarke's generalised gradients and generalised directional derivatives. In [9], the subdifferential formulas for such suprema were derived for an infinite family of convex functions or an infinite family of functions which are directionally differentiable at the considered point uniformly in all directions.

In this paper we study the supremum and the infimum of infinitely many Lipschitz functions defined on a finite dimensional space. The remainder of the paper is organised as follows. After some preliminaries, Sections 3 and 4 are devoted to the discussion of Clarke's generalised gradients and generalised directional derivatives of infinitely many Lipschitz functions. Section 5 give some results ensuring suprema and infima of infinitely many invex Lipschitz functions are invex as well. Finally, in Section 6 applying the previous results, optimality conditions are established for a class of mathematical programming problems under suitable invexity hypotheses.

\section{Received 18th September, 2001}

This research was partially supported by the Natural Science Council of Vietnam. The second author is thankful to the Abdus Salam International Centre for Theoretical Physics, Trieste, for financial support through Grant of Senior Associates.

Copyright Clearance Centre, Inc. Serial-fee code: 0004-9727/02 \$A2.00+0.00. 


\section{Preliminaries}

Let $X$ be a finite dimensional linear space, and let $f$ be a locally Lipschitz function defined on $X$. Following [3], the Clarke generalised directional derivative of the function $f$ at $\bar{x} \in X$, with respect to the direction $d$, is defined as

$$
f^{0}(\bar{x} ; d)=\limsup _{\substack{x \rightarrow \bar{x} \\ t \downarrow 0}} \frac{f(x+t d)-f(x)}{t} .
$$

Then the function $f^{0}(\bar{x} ;$.$) is finite, positively homogeneous and subadditive.$

The following set is called the Clarke generalised gradient of $f$ at $\bar{x}$

$$
\partial f(\bar{x})=\left\{x^{*} \in X^{*}:\left\langle x^{*}, d\right\rangle \leqslant f^{0}(\bar{x} ; d), \forall d \in X\right\},
$$

where $X^{*}$, is the topological dual of $X$. Here, $X^{*}=X$, as $X$ is finite dimension.

Clarke $[3]$ proved that $\partial f(\bar{x})$ is nonempty, convex, compact and

$$
f^{0}(\bar{x} ; d)=\max _{\xi \in \partial f(\bar{x})}\langle\xi, d\rangle \quad(\forall d \in X) .
$$

The set-valued mapping $\partial f($.$) is upper semicontinuous at \bar{x}$. Moreover,

$$
\partial f(\bar{x})=\operatorname{co}\left\{\lim \nabla f\left(x_{i}\right): x_{i} \rightarrow \bar{x}, x_{i} \notin \Omega_{f} \cup S\right\}
$$

where $\Omega_{f}$ stands for the set of nondifferentiable points of $f$ in a neighbourhood $U$ of $\bar{x}$, co indicates the convex hull, and $S$ is any subset of Lebesgue's measure zero in $U$.

The directional derivative of $f$ at $\bar{x}$, with respect to the direction $d$, is the limit

$$
f^{\prime}(\bar{x} ; d)=\lim _{t \downarrow 0} \frac{f(\bar{x}+t d)-f(\bar{x})}{t}
$$

if it exists.

Recall ([3]) that the locally Lipschitz function $f$ is called regular at $\bar{x}$ if there exists the directional derivative $f^{\prime}\left(\bar{x}_{;}\right.$.) and

$$
f^{0}(\bar{x} ; .)=f^{\prime}(\bar{x} ; .)
$$

Following [15], the locally Lipschitz function $f$ is called invex at $\bar{x}$ with respect to a scale function $\omega: X \times X \rightarrow X$ if

$$
f(x)-f(\bar{x}) \geqslant f^{0}(\bar{x} ; \omega(x, \bar{x})) \quad(\forall x \in X),
$$

which is equivalent to the following

$$
f(x)-f(\bar{x}) \geqslant\langle\zeta, \omega(x, \bar{x})\rangle \quad(\forall \zeta \in \partial f(\bar{x}), \forall x \in X)
$$


If (1) or $\left(1^{\prime}\right)$ holds for all $x$ in a subset $U$ containing $\bar{x}$, then $f$ is called invex on $U$ at $\bar{x}$.

Let the locally Lipschitz function $f$ be directionally differentiable at $\bar{x}$ in all directions. It is obvious that

$$
f^{\prime}(\bar{x} ; d) \leqslant f^{0}(\bar{x} ; d) \quad(\forall d) .
$$

Then, $f$ is called weakly invex on $U$ at $\bar{x}$ with respect to a scale function $\omega$ if

$$
f(x)-f(\bar{x}) \geqslant f^{\prime}(\bar{x} ; \omega(x, \bar{x})) \quad(\forall x \in U) .
$$

Thus, if $f$ is invex on $U$ at $\bar{x}$ with respect to $\omega$, then it is weakly invex on $U$ at $\bar{x}$ with respect to $\omega$.

\section{Generalised Gradients}

Let $Q$ be a metrisable compact topological space, and for all $\alpha \in Q$, let $f_{\alpha}$ be a real-valued function defined on $\mathbb{R}^{n}$. From now on we define the following functions

$$
f(x)=\sup _{\alpha \in Q} f_{\alpha}(x), \quad g(x)=\inf _{\alpha \in Q} f_{\alpha}(x) .
$$

Let $U$ be an open subset of $\mathbb{R}^{n}$ containing $\bar{x}$. Assume that for all $\alpha \in Q$, the function $f_{\alpha}$ is Lipschitzian on $U$ with the same Lipschitz constant $K$. Due to Rademacher's theorem, $f_{\alpha}$ is differentiable almost anywhere in $U$ with respect to Lebesgue's measure on $\mathbb{R}^{n}$. In view of the compactness of $Q, f(x)$ and $g(x)$ are finite at each $x \in \mathbb{R}^{n}$.

Donote by $\Omega_{\alpha}$ the set of points of $U$ at which $f_{\alpha}$ is nondifferentiable. Then $\Omega_{\alpha}$ is of Lebesgue's measure zero.

Define the following sets

$$
\begin{aligned}
& Q_{0}=\left\{\alpha \in Q: f_{\alpha}(\bar{x})=\sup _{\beta \in Q} f_{\beta}(\bar{x})\right\}, \\
& Q_{1}=\left\{\alpha \in Q: f_{\alpha}(\bar{x})=\inf _{\beta \in Q} f_{\beta}(\bar{x})\right\} .
\end{aligned}
$$

Note that the aforementional functions $f$ and $g$ are Lipschitzian on $U$.

A theorem on Clarke's generalised gradient of the function $f$ defined above can be stated as follows

THEOREM 3.1. Assume that the mapping $\alpha \mapsto f_{\alpha}(\bar{x})$ is continuous, and the set-valued mapping $(\alpha, x) \mapsto \partial f_{\alpha}(x)$ is upper semicontinuous at $(\alpha, \bar{x})$ for all $\alpha \in Q_{0}$. Then

$$
\partial f(\bar{x}) \subset \operatorname{co}\left(\bigcup_{\alpha \in Q_{0}} \partial f_{\alpha}(\bar{x})\right)
$$


PROOF: We begin by establishing the following

$$
\partial f(\bar{x}) \subset \overline{c 0}\left(\bigcup_{\alpha \in Q_{0}} \partial f_{\alpha}(\bar{x})\right),
$$

where $\overline{c o}$ indicates the closed convex hull.

In view of $[3$, Theorem 2.8 .6$]$, one gets

$$
\partial f(\bar{x}) \subset \overline{c 0}\left\{\lim _{i \rightarrow \infty} \nabla f_{\alpha_{i}}\left(x_{i}\right): x_{i} \rightarrow \bar{x}, x_{i} \notin S_{1}, x_{i} \notin \Omega_{\alpha_{i}}, \alpha_{i} \in Q, f_{\alpha_{i}}(\bar{x}) \rightarrow f(\bar{x})\right\},
$$

where $S_{1}$ is an arbitrary subset of $U$ with Lebesgue's measure zero.

Define the set

$$
A=\left\{\lim _{i \rightarrow \infty} \nabla f_{\alpha_{i}}\left(x_{i}\right): x_{i} \rightarrow \bar{x}, x_{i} \notin S_{1}, x_{i} \notin \Omega_{\alpha_{i}}, \alpha_{i} \in Q, f_{\alpha_{i}}(\bar{x}) \rightarrow f(\bar{x})\right\} .
$$

To obtain (4), it is sufficient to verify that

$$
A \subset \bigcup_{\alpha \in Q_{0}} \partial f_{\alpha}(\bar{x})
$$

Taking $\xi \in A$, we have $\xi=\lim _{i \rightarrow \infty} \nabla f_{\alpha_{i}}\left(x_{i}\right)$ in which $x_{i} \rightarrow \bar{x}, x_{i} \notin S_{1}, x_{i} \notin \Omega_{\alpha_{i}}$, $\alpha_{i} \in Q, f_{\alpha_{i}}(\bar{x}) \rightarrow f(\bar{x})$.

Since $Q$ is a metrisable compact topological space, there exists a subsequence $\left\{\alpha_{i_{k}}\right\}$ of the sequence $\left\{\alpha_{i}\right\}$ such that $\alpha_{i_{k}} \rightarrow \alpha_{0} \in Q$ as $k \rightarrow \infty$. In view of the continuity of the mapping $\alpha \mapsto f_{\alpha}(\bar{x})$, we obtain that $f_{\alpha_{i_{k}}}(\bar{x}) \rightarrow f_{\alpha_{0}}(\bar{x})$ as $k \rightarrow \infty$. Moreover, we also get that $f_{\alpha_{i_{k}}}(\bar{x}) \rightarrow f(\bar{x})$ as $k \rightarrow \infty$. Hence, $f_{\alpha_{0}}(\bar{x})=f(\bar{x})$, which means that $\alpha_{0} \in Q_{0}$.

Observing $\nabla f_{\alpha_{i_{k}}}\left(x_{i_{k}}\right) \in \partial f_{\alpha_{i_{k}}}\left(x_{i_{k}}\right)(\forall k)$, by virtue of the upper semicontinuity of the mapping $(\alpha, x) \mapsto \partial f_{\alpha}(x)$, we contend that

$$
\xi=\lim _{k \rightarrow \infty} \nabla f \alpha_{i_{k}}\left(x_{i_{k}}\right) \in \partial f_{\alpha_{0}}(\bar{x})
$$

which gives (5).

Setting $f_{0}:=\sup _{\alpha \in Q} f_{\alpha}(\bar{x})$, in view of the compactness of $Q, f_{0}$ is finite. We define the set $P_{0}:=\left\{\alpha \cdot \in Q: f_{\alpha}(\bar{x})<f_{0}\right\}$. In view of the continuity of the mapping $\alpha \mapsto f_{\alpha}(\bar{x})$, the set $P_{0}$ is an open subset of $Q$, and hence $Q \backslash P_{0}$ is a closed subset of $Q$. Since $Q_{0}=Q \backslash P_{0}$ and $Q$ is compact, it follows that $Q_{0}$ is compact.

Observing that $\left(\mathbb{R}^{n}\right)^{*}=\mathbb{R}^{n}$, one gets $\bigcup_{\alpha \in Q_{0}} \partial f_{\alpha}(\bar{x}) \subset \mathbb{R}^{n}$. Making use of the compactness of $Q_{0}$ and the upper semicontinuity of the mapping $\alpha \mapsto \partial f_{\alpha}(\bar{x})$, we claim that the set $\bigcup_{\alpha \in Q_{0}} \partial f_{\alpha}(\bar{x})$ is compact in $\mathbb{R}^{n}$ (see, for example, [2, p. 116]). 
It is well known that the convex hull of a compact set in $\mathbb{R}^{n}$ is also compact. Hence, co $\left(\bigcup_{\alpha \in Q_{0}} \partial f_{\alpha}(\bar{x})\right)$ is compact, and thus co $\left(\bigcup_{\alpha \in Q_{0}} \partial f_{\alpha}(\bar{x})\right)$ is closed. Moreover, according to the convex analysis,

$$
\overline{\operatorname{co}}\left(\bigcup_{\alpha \in Q_{0}} \partial f_{\alpha}(\bar{x})\right)=\overline{\operatorname{co}\left(\bigcup_{\alpha \in Q_{0}} \partial f_{\alpha}(\bar{x})\right)}=\operatorname{co}\left(\bigcup_{\alpha \in Q_{0}} \partial f_{\alpha}(\bar{x})\right),
$$

which together with (4) implies (3). The proof is complete.

For calculating Clarke's generalised gradient of the function $g$ defined above we have the following

THEOREM 3.2. Suppose that the mapping $\alpha \mapsto f_{\alpha}(\bar{x})$ is continuous, and the set-valued mapping $(\alpha, x) \mapsto-\partial f_{\alpha}(x)$ is upper semicontinuous at $(\alpha, \bar{x})$ for all $\alpha \in Q_{1}$. Then,

$$
\partial g(\bar{x}) \subset \operatorname{co}\left(\bigcup_{\alpha \in Q_{1}} \partial f_{\alpha}(\bar{x})\right)
$$

Proof: Observe that

$$
\begin{aligned}
-g(\bar{x}) & =-\inf _{\alpha \in Q} f_{\alpha}(\bar{x})=\sup _{\beta \in Q}\left(-f_{\beta}(\bar{x})\right), \\
Q_{1} & =\left\{\alpha \in Q:-f_{\alpha}(\bar{x})=\sup _{\beta \in Q}\left(-f_{\beta}(\bar{x})\right)\right\} .
\end{aligned}
$$

Due to the hypotheses, the mapping $\alpha \mapsto-f_{\alpha}(\bar{x})$ is continuous, and the set-valued mapping $(\alpha, x) \mapsto \partial\left(-f_{\alpha}\right)(x)$ is upper semicontinuous at $(\alpha, \bar{x})$ for all $\alpha \in Q_{1}$. Applying Theorem 3.1 to the functions $-f_{\alpha}(\alpha \in Q)$ we obtain

$$
\partial(-g)(\bar{x}) \subset \operatorname{co}\left(\bigcup_{\alpha \in Q_{1}} \partial\left(-f_{\alpha}\right)(\bar{x})\right)
$$

It follows from the nonsmooth analysis in [3] that

$$
\begin{aligned}
-\partial g(\bar{x}) & =\partial(-g)(\bar{x}) \subset \operatorname{co}\left(\bigcup_{\alpha \in Q_{1}} \partial\left(-f_{\alpha}\right)(\bar{x})\right) \\
& =\operatorname{co}\left(-\bigcup_{\alpha \in Q_{1}} \partial f_{\alpha}(\bar{x})\right) \\
& =-\operatorname{co}\left(\bigcup_{\alpha \in Q_{1}} \partial f_{\alpha}(\bar{x})\right) .
\end{aligned}
$$

Consequently,

$$
\partial g(\bar{x}) \subset \operatorname{co}\left(\bigcup_{\alpha \in Q_{1}} \partial f_{\alpha}(\bar{x})\right)
$$




\section{Generalised Directional Derivatives}

THEOREM 4.1. Assume that the mapping $\alpha \mapsto f_{\alpha}(\bar{x})$ is continuous, and the set-valued mapping $(\alpha, x) \mapsto \partial f_{\alpha}(x)$ is upper semicontinuous at $(\alpha, \bar{x})$ for all $\alpha \in Q_{0}$. Then,

$$
f^{0}(\bar{x} ; d) \leqslant \sup _{\alpha \in Q_{0}} f_{\alpha}^{0}(\bar{x} ; d) \quad(\forall d)
$$

Moreover, if for all $\alpha \in Q_{0}, f_{\alpha}$ is regular at $\bar{x}$, then $f$ is also regular at $\bar{x}$, and

$$
\begin{aligned}
f^{\prime}(\bar{x} ; d) & =\sup _{\alpha \in Q_{0}} f_{\alpha}^{\prime}(\bar{x} ; d) \quad(\forall d), \\
\partial f(\bar{x}) & =\operatorname{co}\left(\bigcup_{\alpha \in Q_{0}} \partial f_{\alpha}(\bar{x})\right) .
\end{aligned}
$$

Proof: We invoke Theorem 3.1 to deduce that

$$
\partial f(\bar{x}) \dot{c} \operatorname{co}\left(\bigcup_{\alpha \in Q_{0}} \partial f_{\alpha}(\bar{x})\right)
$$

Hence, for every $d \in \mathbb{R}^{n}$,

$$
\begin{aligned}
f^{0}(\bar{x} ; d) & =\max _{\xi \in \partial f(\bar{x})}\langle\xi, d\rangle \leqslant \max \left\{\langle\xi, d\rangle: \xi \in \operatorname{co}\left(\bigcup_{\alpha \in Q_{0}} \partial f_{\alpha}(\bar{x})\right)\right\} \\
& =\left\langle\xi_{0}, d\right\rangle \quad\left(\text { for some } \xi_{0} \in \operatorname{co}\left(\bigcup_{\alpha \in Q_{0}} \partial f_{\alpha}(\bar{x})\right)\right) \\
& =\left\langle\sum_{i=1}^{m} \lambda_{i} \xi_{\alpha_{i}}, d\right\rangle,
\end{aligned}
$$

for some $\xi_{\alpha_{i}} \in \partial f_{\alpha_{i}}(\bar{x}), \lambda_{i} \geqslant 0, i=1, \ldots, m, \alpha_{i} \in Q_{0}, \sum_{i=1}^{m} \lambda_{i}=1$

$$
\begin{aligned}
\left\langle\sum_{i=1}^{m} \lambda_{i} \xi_{\alpha_{i}}, d\right\rangle & \leqslant \sum_{i=1}^{m} \lambda_{i} \sup _{\alpha \in Q_{0}} \max _{\xi_{\alpha} \in \partial f_{\alpha}(\bar{x})}\left\langle\xi_{\alpha}, d\right\rangle \\
& =\sup _{\alpha \in Q_{0}} \max _{\xi_{\alpha} \in \partial f_{\alpha}(\bar{x})}\left\langle\xi_{\alpha}, d\right\rangle \\
& =\sup _{\alpha \in Q_{0}} f_{\alpha}^{0}(\bar{x} ; d) .
\end{aligned}
$$

Assume now that for all $\alpha \in Q_{0}, f_{\alpha}$ is regular at $\bar{x}$. By (7), it follows that for every $d \in \mathbb{R}^{n}$,

$$
\limsup _{t \downarrow 0} \frac{f(\bar{x}+t d)-f(\bar{x})}{t} \leqslant f^{0}(\bar{x} ; d) \leqslant \sup _{\alpha \in Q_{0}} f_{\alpha}^{\prime}(\bar{x} ; d) .
$$


On the other hand, for each $\alpha \in Q_{0}$,

$$
\liminf _{t \downarrow 0} \frac{f(\bar{x}+t d)-f(\bar{x})}{t} \geqslant \liminf _{t \downarrow 0} \frac{f_{\alpha}(\bar{x}+t d)-f_{\alpha}(\bar{x})}{t}=f_{\alpha}^{\prime}(\bar{x} ; d),
$$

which implies that

$$
\liminf _{t \downarrow 0} \frac{f(\bar{x}+t d)-f(\bar{x})}{t} \geqslant \sup _{\alpha \in Q_{0}} f_{\alpha}^{\prime}(\bar{x} ; d) .
$$

Combining (11) and (12) yields that the directional derivative of $f$ at $\bar{x}$ exists

$$
f^{\prime}(\bar{x} ; d)=\lim _{t \downarrow 0} \frac{f(\bar{x}+t d)-f(\bar{x})}{t}=f^{0}(\bar{x} ; d),
$$

and

$$
f^{\prime}(\bar{x} ; d)=\sup _{\alpha \in Q_{0}} f_{\alpha}^{\prime}(\bar{x} ; d), \quad \text { for all } d .
$$

It should be noted that in view of (10), one gets

$$
\begin{aligned}
f^{\prime}(\bar{x} ; d) & =\max _{\xi \in \partial f(\bar{x})}\langle\xi, d\rangle \leqslant \max _{\xi \in \operatorname{co}}\left(\bigcup_{\alpha \in Q_{0}} \partial f_{\alpha}(\bar{x})\right) \\
& \leqslant \sup _{\alpha \in Q_{0}} \max _{\xi_{\alpha} \in \partial f_{\alpha}(\bar{x})}\left\langle\xi_{\alpha}, d\right\rangle \\
& =\sup _{\alpha \in Q_{0}} f_{\alpha}^{\prime}(\bar{x} ; d) \quad(\forall d),
\end{aligned}
$$

which together with (13) yields that

$$
\begin{aligned}
f^{\prime}(\bar{x} ; d) & =\max _{\xi \in \partial f(\bar{x})}\langle\xi, d\rangle=\max _{\xi \in \mathrm{co}}\left(\bigcup_{\alpha \in Q_{0}} \partial f_{\alpha}(\bar{x})\right) \\
& =\sup _{\alpha \in Q_{0}} f_{\alpha}^{\prime}(\bar{x} ; d) .
\end{aligned}
$$

Because of the closeness and the convexity of $\partial f(\bar{x})$ and $\operatorname{co}\left(\bigcup_{\alpha \in \alpha_{0}} \partial f_{\alpha}(\bar{x})\right)$, from (14) we claim (see, for example, [3, Proposition 2.14]) that

$$
\partial f(\bar{x})=\operatorname{co}\left(\bigcup_{\alpha \in Q_{0}} \partial f_{\alpha}(\bar{x})\right)
$$

This concludes the proof.

We now turn to the function $g$. 
THEOREM 4.2. Assume that the mapping $\alpha \mapsto f_{\alpha}(\bar{x})$ is continuous, and the setvalued mapping $(\alpha, x) \mapsto-\partial f_{\alpha}(x)$ is upper semicontinuous at $(\alpha, \bar{x})$ for all $\alpha \in Q_{1}$. Suppose, in addition, that for all $\alpha \in Q_{1}$, the function $-f_{\alpha}$ is regular at $\bar{x}$. Then, $-g$ is regular at $\bar{x}$, and

$$
\begin{aligned}
g^{\prime}(\bar{x} ; d) & =\inf _{\alpha \in Q_{1}} f_{\alpha}^{\prime}(\bar{x} ; d) \quad(\forall d), \\
\partial g(\bar{x}) & =c o\left(\bigcup_{\alpha \in Q_{1}} \partial f_{\alpha}(\bar{x})\right)
\end{aligned}
$$

Proof: According to the hypotheses, the mapping $\alpha \mapsto-f_{\alpha}(\bar{x})$ is continuous, and the set-valued mapping $(\alpha, x) \mapsto \partial\left(-f_{\alpha}\right)(x)$ is upper semicontinuous. At this point we remark that

$$
\begin{aligned}
-g(x) & =-\inf _{\alpha \in Q} f_{\alpha}(x)=\sup _{\alpha \in Q}\left(-f_{\alpha}(x)\right), \\
Q_{1} & =\left\{\alpha \in Q:-f_{\alpha}(\bar{x})=\sup _{\beta \in Q}\left(-f_{\beta}(\bar{x})\right)\right\} .
\end{aligned}
$$

Note that $f_{\alpha}$ is also directionally differentiable at $\bar{x}$ in all directions.

Applying Theorem 4.1 to the functions $-f_{\alpha}\left(\alpha \in Q_{1}\right)$ at $\bar{x}$, we claim that the function $-g$ is regular at $\bar{x}$, and

$$
\begin{aligned}
(-g)^{0}(\bar{x} ; d) & =-g^{\prime}(\bar{x} ; d)=\sup _{\alpha \in Q_{1}}\left(-f_{\alpha}\right)^{\prime}(\bar{x} ; d) \\
& =-\inf _{\alpha \in Q_{1}} f_{\alpha}^{\prime}(\bar{x} ; d) \quad(\forall d) .
\end{aligned}
$$

Thus, $g$ is directionally differentiable at $\bar{x}$ in all directions, and

$$
g^{\prime}(\bar{x} ; d)=\inf _{\alpha \in Q_{1}} f_{\alpha}^{\prime}(\bar{x} ; d) \quad(\forall d)
$$

We now invoke Theorem 4.1 to deduce that

$$
\partial(-g)(\bar{x})=\operatorname{co}\left(\bigcup_{\alpha \in Q_{1}} \partial\left(-f_{\alpha}\right)(\bar{x})\right)=-\operatorname{co}\left(\bigcup_{\alpha \in Q_{1}} \partial f_{\alpha}(\bar{x})\right) .
$$

Consequently,

$$
\partial g(\bar{x})=-\partial(-g)(\bar{x})=\operatorname{co}\left(\bigcup_{\alpha \in Q_{1}} \partial f_{\alpha}(\bar{x})\right)
$$




\section{INVEXITY}

We are now in a position to formulate conditions ensuring the supremum of Lipschitz invex functions is invex.

THEOREM 5.1. Assume that the mapping $\alpha \mapsto f_{\alpha}(\bar{x})$ is continuous the mappings $\alpha \mapsto f_{\alpha}^{0}(\bar{x} ; d)$ for all $d$ is upper semicontinuous, and the set-valued mapping $(\alpha, x) \mapsto \partial f_{\alpha}(x)$ is upper semicontinuous at $(\alpha, \bar{x})\left(\forall \alpha \in Q_{0}\right)$. Suppose, furthermore, that for all $\alpha \in Q_{0}, f_{\alpha}$ is invex on $U$ at $\bar{x}$ with respect to the same scale function $\omega: U \times U \rightarrow \mathbb{R}^{n}$. Then, the function $f$ defined by (2) is invex on $U$ at $\bar{x}$ with respect to the scale function $\omega$.

Proof: Assume the contrary, that the conclusion is false. Then there exists $x_{1} \in$ $U$ such that

$$
f\left(x_{1}\right)-f(\bar{x})<f^{0}\left(\bar{x} ; \omega\left(x_{1}, \bar{x}\right)\right) .
$$

Due to Theorem 4.1, it follows from (17) that

$$
\begin{aligned}
\sup _{\alpha \in Q_{0}} f_{\alpha}\left(x_{1}\right)-\sup _{\alpha \in Q_{0}} f_{\alpha}(\bar{x}) & \leqslant \sup _{\alpha \in Q} f_{\alpha}\left(x_{1}\right)-\sup _{\alpha \in Q} f_{\alpha}(\bar{x}) \\
& <f^{0}\left(\bar{x} ; \omega\left(x_{1}, \bar{x}\right)\right) \\
& \leqslant \sup _{\alpha \in Q_{0}} f_{\alpha}^{0}\left(\bar{x} ; \omega\left(x_{1}, \bar{x}\right)\right)
\end{aligned}
$$

In view of the compactness of $Q_{0}$ and the upper semicontinuity of the mapping $\alpha \mapsto f_{\alpha}^{0}\left(\bar{x} ; \omega\left(x_{1}, \bar{x}\right)\right)$, there exists $\alpha_{0} \in Q_{0}$ such that

$$
\sup _{\alpha \in Q_{0}} f_{\alpha}^{0}\left(\bar{x} ; \omega\left(x_{1}, \bar{x}\right)\right)=f_{\alpha_{0}}^{0}\left(\bar{x} ; \omega\left(x_{1}, \bar{x}\right)\right) \text {. }
$$

Combining (18) and (19) yields that

$$
\sup _{\alpha \in Q_{0}}\left[f_{\alpha}\left(x_{1}\right)-f_{\alpha}(\bar{x})\right]<f_{\alpha_{0}}^{0}\left(\bar{x} ; \omega\left(x_{1}, \bar{x}\right)\right) .
$$

Consequently,

$$
f_{\alpha_{0}}\left(x_{1}\right)-f_{\alpha_{0}}(\bar{x})<f_{\alpha_{0}}^{0}\left(\bar{x} ; \omega\left(x_{1}, \bar{x}\right)\right),
$$

which means that $f_{\alpha_{0}}$ is not invex on $U$ at $\bar{x}$ with respect to $\omega$.

This contradicts the hypothesis.

Theorem 5.2. Assume that the mapping $\alpha \mapsto f_{\alpha}(\bar{x})$ is continuous, and the set-valued mapping $(\alpha, x) \mapsto \partial f_{\alpha}(x)$ is upper semicontinuous at $(\alpha, \bar{x})$ for all $\alpha \in Q_{0}$. Suppose, in addition, that for all $\alpha \in Q_{0}, f_{\alpha}$ is regular at $\bar{x}$ and invex on $U$ at $\bar{x}$ with 
respect to the same scale function $\omega: U \times U \rightarrow \mathbb{R}^{n}$. Then the function $f$ defined by (2) is invex on $U$ at $\bar{x}$ with respect to $\omega$.

Proof: Since for all $\alpha \in Q_{0}, f_{\alpha}$ is regular at $\bar{x}$ and invex on $U$ at $\bar{x}$ with respect to the same scale function $\omega$, it follows that for every $x \in U$,

$$
f_{\alpha}(x) \geqslant f_{\alpha}^{\prime}(\bar{x} ; \omega(x, \bar{x}))+f_{\alpha}(\bar{x})
$$

which implies that for every $\alpha \in Q_{0}$,

$$
\sup _{\alpha \in Q} f_{\alpha}(x) \geqslant \sup _{\alpha \in Q_{0}} f_{\alpha}(x) \geqslant f_{\alpha}^{\prime}(\bar{x} ; \omega(x, \bar{x}))+f_{\alpha}(\bar{x}) .
$$

Hence,

$$
\sup _{\alpha \in Q} f_{\alpha}(x) \geqslant \sup _{\alpha \in Q_{0}} f_{\alpha}^{\prime}(\bar{x} ; \omega(x, \bar{x}))+\sup _{\alpha \in Q_{0}} f_{\alpha}(\bar{x}) .
$$

We invoke Theorem 4.1 to deduce that for every $x \in U$,

$$
f^{\prime}(\bar{x} ; \omega(x, \bar{x}))=\sup _{\alpha \in Q_{0}} f_{\alpha}^{\prime}(\bar{x} ; \omega(x, \bar{x})) .
$$

Substituting (21) into (20) yields that

$$
f(x)-f(\bar{x}) \geqslant f^{\prime}(\bar{x} ; \omega(x, \bar{x})),
$$

which gives the assertion.

Denote by $B(\bar{x} ; \delta)$ the open ball of radius $\delta>0$ around $\bar{x}$.

THEOREM 5.3. Assume that for all $x \in U, x \neq \bar{x}$, the mapping $\alpha \mapsto f_{\alpha}(x)$ is lower semicontinuous, the mapping $\alpha \mapsto f_{\alpha}(\bar{x})$ is continuous, and the set-valued mapping $(\alpha, x) \mapsto-\partial f_{\alpha}(\bar{x})$ is upper semicontinuous at $(\alpha, \bar{x}) \quad\left(\forall \alpha \in Q_{1}\right)$. Suppose, furthermore, that for all $\alpha \in Q_{1},-f_{\alpha}$ is regular at $\bar{x}$, and $f_{\alpha}$ is weakly invex on $U$ at $\bar{x}$ with respect to the same scale function $\omega$. Then there exists a number $\delta>0$ such that the function $g$ defined by (2) is weakly invex on $B(\bar{x} ; \delta) \cap U$ at $\bar{x}$ with respect to $\omega$.

Proof: We first observe that for all $\alpha \in Q_{1}, f_{\alpha}$ is directionally differentiable at $\bar{x}$ in all directions. According to Theorem 4.2, $g$ is also directionally differentiable at $\bar{x}$ in all directions.

Assume now the contrary, that the conclusion is false. Then for every $\delta>0$ there exists $x_{1} \in B(\bar{x} ; \delta) \cap U$ such that

$$
g\left(x_{1}\right)-g(\bar{x})<g^{\prime}\left(\bar{x} ; \omega\left(x_{1}, \bar{x}\right)\right) .
$$


Taking account of Theorem 4.2 , one gets

$$
g^{\prime}(\bar{x} ; \omega(x, \bar{x}))=\inf _{\alpha \in Q_{1}} f_{\alpha}^{\prime}\left(\bar{x} ; \omega\left(x_{1}, \bar{x}\right)\right) .
$$

Substituting (23) into (22) yields that

$$
\inf _{\alpha \in Q} f_{\alpha}\left(x_{1}\right)<\inf _{\alpha \in Q_{1}} f_{\alpha}^{\prime}\left(\bar{x} ; \omega\left(x_{1}, \bar{x}\right)\right)+\inf _{\alpha \in Q} f_{\alpha}(\bar{x}),
$$

which implies that

$$
\inf _{\alpha \in Q} f_{\alpha}\left(x_{1}\right)<\inf _{\alpha \in Q_{1}}\left[f_{\alpha}^{\prime}\left(\bar{x} ; \omega\left(x_{1}, \bar{x}\right)\right)+f_{\alpha}(\bar{x})\right] .
$$

In view of the lower semicontinuity of the mapping $\alpha \mapsto f_{\alpha}\left(x_{1}\right)$, there exists $\beta \in Q$ such that

$$
\inf _{\alpha \in Q} f_{\alpha}\left(x_{1}\right)=f_{\beta}\left(x_{1}\right)
$$

whence,

$$
f_{\beta}\left(x_{1}\right)<\inf _{\alpha \in Q_{1}}\left[f_{\alpha}^{\prime}\left(\bar{x} ; \omega\left(x_{1}, \bar{x}\right)\right)+f_{\alpha}(\bar{x})\right] .
$$

We now show that $\beta \in Q_{1}$.

Indeed, if $\beta \notin Q_{1}$, then for a fixed $\alpha \in Q_{1}$,

$$
f_{\beta}(\bar{x})-f_{\alpha}(\bar{x})=\mu>0 .
$$

Because of the continuity of $f_{\alpha}$, there exists $\delta_{1}>0$ such that for every $x \in B\left(\bar{x} ; \delta_{1}\right) \cap U$,

$$
f_{\alpha}(x) \leqslant f_{\alpha}(\bar{x})+\frac{\mu}{3} .
$$

In view of the continuity of $f_{\beta}$, there exists $\delta_{2}>0$ such that for every $x \in B\left(\bar{x} ; \delta_{2}\right) \cap U$,

$$
f_{\beta}(\bar{x})-\frac{\mu}{3} \leqslant f_{\beta}(x) \text {. }
$$

Taking $\delta=\min \left\{\delta_{1}, \delta_{2}\right\}$, it follows from (26)-(28) that for every $x \in B(\bar{x} ; \delta) \cap U$,

$$
f_{\alpha}(x) \leqslant f_{\alpha}(\bar{x})+\frac{\mu}{3}<f_{\beta}(\bar{x})-\frac{\mu}{3} \leqslant f_{\beta}(x),
$$

which implies that

$$
f_{\alpha}\left(x_{1}\right)<f_{\beta}\left(x_{1}\right)
$$


This conflicts with (24). Consequently, $\beta \in Q_{1}$.

On the other hand, by (25) we obtain

$$
f_{\beta}\left(x_{1}\right)<f_{\alpha}^{\prime}\left(\bar{x} ; \omega\left(x_{1}, \bar{x}\right)\right)+f_{\alpha}(\bar{x}) \quad\left(\forall \alpha \in Q_{1}\right),
$$

which leads to the following

$$
f_{\beta}\left(x_{1}\right)<f_{\beta}^{\prime}\left(\bar{x} ; \omega\left(x_{1}, \bar{x}\right)\right)+f_{\beta}(\bar{x}) .
$$

This means that $f_{\beta}$ is not weakly invex on $U$ at $\bar{x}$ with respect to $\omega$, which contradicts the hypothesis.

Corollary 5.1. Assume all the hypotheses of Theorem 5.3 are satisfied. Suppose, furthermore, that the function $g$ defined by (2) is regular at $\bar{x}$. Then there exists a number $\delta>0$ such that $g$ is invex on $B(\bar{x} ; \delta) \cap U$ at $\bar{x}$ with respect to $\omega$.

\section{Applications}

Let $Q$ and $B$. be metrisable compact topological spaces, and let $f_{\alpha}(\alpha \in Q)$ and $g_{\beta}$ $(\beta \in B)$ be real-valued functions defined on $\mathbb{R}^{n}$. In this section we shall be concerned with the following problem

$$
\text { (P) } \begin{cases}\text { minimise } & \inf _{\alpha \in Q} f_{\alpha}(x), \\ \text { subject to } & \sup _{\beta \in B} g_{\beta}(x) \leqslant 0 .\end{cases}
$$

In [13] Luu and Oettli studied a similar problem in rather general setting.

Define the following sets

$$
\begin{aligned}
& Q_{0}(\bar{x})=\left\{\alpha \in Q: f_{\alpha}(\bar{x})=\inf _{\gamma \in Q} f_{\gamma}(\bar{x})\right\}, \\
& B_{0}(\bar{x})=\left\{\beta \in B: g_{\beta}(\bar{x})=\sup _{\gamma \in B} g_{\gamma}(\bar{x})\right\} .
\end{aligned}
$$

We are now in a position to formulate a necessary optimallity conditions for Problem (P).

Theorem 6.1. Let $\bar{x}$ be a local minimiser of Problem (P). Assume that the functions $f_{\alpha}(\alpha \in Q)$ and $g_{\beta}(\beta \in B)$ are Lipschitzian on the same open set $U$ containing $\bar{x}$ with the same Lipschitz constants $K_{1}$ and $K_{2}$, respectively. Suppose, in addition, that the following two conditions are satisfied.

(a) The mapping $\alpha \mapsto f_{\alpha}(\bar{x})$ is continuous, and the set-valued mapping $(\alpha, x) \mapsto-\partial f_{\alpha}(x)$ is upper semicontinuous at $(\alpha, \bar{x})$ for all $\alpha \in Q_{0}(\bar{x})$;

(b) The mapping $\beta \mapsto g_{\beta}(\bar{x})$ is continuous, and the set-valued mapping $(\beta, x) \mapsto \partial g_{\beta}(x)$ is upper semicontinuous at $(\beta, \bar{x})$ for all $\beta \in B_{0}(\bar{x})$. 
Then, there exist $\bar{\sigma} \geqslant 0, \bar{\chi} \geqslant 0, \alpha_{i} \in Q_{0}(\bar{x}), \bar{\lambda}_{\alpha_{i}} \geqslant 0, i=1, \ldots, m$, with $\sum_{i=1}^{m} \bar{\lambda}_{\alpha_{i}}=1$, and $\beta_{j} \in B_{0}(\bar{x}), \bar{\mu}_{\beta_{j}} \geqslant 0, j=1, \ldots, \ell$ with $\sum_{j=1}^{\ell} \bar{\mu}_{\beta_{j}}=1, \bar{\sigma}$ and $\bar{\chi}$ are not both equal to zero, such that

$$
\begin{gathered}
0 \in \bar{\sigma} \sum_{i=1}^{m} \bar{\lambda}_{\alpha_{i}} \partial f_{\alpha_{i}}(\bar{x})+\bar{\chi} \sum_{j=1}^{\ell} \bar{\mu}_{\beta_{j}} \partial g_{\beta_{j}}(\bar{x}) \\
\bar{\chi} \sup _{\beta \in B_{0}(\bar{x})} g_{\beta}(\bar{x})=0 .
\end{gathered}
$$

Moreover, assume the following conditions hold

(i) There exists $\widehat{x} \in U$ such that $\sup _{\beta \in B} g_{\beta}(\widehat{x})<0$;

(ii) For all $\beta \in B_{0}(\bar{x})$, the function $g_{\beta}$ is regular at $\bar{x}$ and invex on $U$ at $\bar{x}$ with respect to the same scale functions $\omega$.

Then $\bar{\sigma}>0$, and it can be taken $\bar{\sigma}=1$.

Proof: Let $\tilde{f}(x):=\inf _{\alpha \in Q} f_{\alpha}(x)$ and $\tilde{g}(x):=\sup _{\beta \in B} g_{\beta}(x)$. Then, the functions $\tilde{f}$ and $\tilde{g}$ are Lipschitzian on $U$ with the Lipschitz constant $K_{1}$ and $K_{2}$, respectively. Due to [3, Theorem 6.1.1], there exist Lagrange multipliers $\bar{\sigma} \geqslant 0$ and $\bar{\chi} \geqslant 0$, not both zero, such that

$$
\begin{gathered}
0 \in \bar{\sigma} \partial \tilde{f}(\bar{x})+\bar{\chi} \partial \widetilde{g}(\bar{x}), \\
\bar{\chi} \sup _{\beta \in B} g_{\beta}(\bar{x})=0 .
\end{gathered}
$$

Observe that (32) leads to (30).

Applying Theorem 3.2 to the function $\tilde{f}$ and Theorem 3.1 to the function $\tilde{g}$, we claim that

$$
\begin{aligned}
& \partial \tilde{f}(\bar{x}) \subset \operatorname{co}\left(\bigcup_{\alpha \in Q_{0}(\bar{x})} \partial f_{\alpha}(\bar{x})\right), \\
& \partial \tilde{g}(\bar{x}) \subset \operatorname{co}\left(\bigcup_{\beta \in B_{0}(\bar{x})} \partial g_{\beta}(\bar{x})\right) .
\end{aligned}
$$

Substituting (33) and (34) into (31) yields that

$$
0 \in \bar{\sigma} \operatorname{co}\left(\bigcup_{\alpha \in Q_{0}(\bar{x})} \partial f_{\alpha}(\bar{x})\right)+\bar{\chi} \operatorname{co}\left(\bigcup_{\beta \in B_{0}(\bar{x})} \partial g_{\beta}(\bar{x})\right)
$$


which implies that there exist $\alpha_{i} \in Q_{0}(\bar{x}), \bar{\lambda}_{\alpha_{i}} \geqslant 0, i=1, \ldots, m$, with $\sum_{i=1}^{m} \lambda_{\alpha_{i}}=1$, and $\beta_{j} \in B_{0}(\bar{x}), \bar{\mu}_{\beta_{j}} \geqslant 0, j=1, \ldots, \ell$, with $\sum_{j=1}^{\ell} \bar{\mu}_{\beta_{j}}=1$ such that

$$
0 \in \bar{\sigma} \sum_{i=1}^{m} \bar{\lambda}_{\alpha_{i}} \partial f_{\alpha_{i}}(\bar{x})+\bar{\chi} \sum_{j=1}^{\ell} \bar{\mu}_{\beta_{j}} \partial g_{\beta_{j}}(\bar{x}) .
$$

For the final assertion of the theorem, we should show that $\bar{\sigma}>0$. Suppose the contrary, that $\bar{\sigma}=0$. Since $\bar{\sigma}$ and $\bar{\chi}$ are not both equal to zero, it follows that $\bar{\chi}>0$, and (29) becomes

$$
0 \in \bar{\chi} \sum_{j=1}^{\ell} \bar{\mu}_{\beta_{j}} \partial g_{\beta_{j}}(\bar{x}),
$$

which implies that there exist $\bar{\eta}_{j} \in \partial g_{\beta_{j}}(\bar{x})(j=1, \ldots, \ell)$ such that

$$
\sum_{j=1}^{\ell} \bar{\mu}_{\beta_{j}} \bar{\eta}_{j}=0
$$

Define the function

$$
G(x)=\bar{\chi} \sup _{\beta \in B} g_{\beta}(x)
$$

From assumption (i) we can see that

$$
G(\widehat{x})<0 .
$$

Since for $\beta \in B_{0}(\bar{x})$, the function $g_{\beta}$ is regular at $\bar{x}$ and invex on $U$ at $\bar{x}$ with respect to the same scale function $\omega$, it follows from Theorem 4.1 and 5.2 that the function $\tilde{g}$ is regular at $\bar{x}$ and invex on $U$ at $\bar{x}$, that is

$$
\tilde{g}(x)-\tilde{g}(\bar{x}) \geqslant \tilde{g}^{\prime}(\bar{x} ; \omega(x, \bar{x})) \quad(\forall x \in U) .
$$

Taking account of Theorem 4.1 , we get

$$
\partial \widetilde{g}(\bar{x})=\operatorname{co}\left(\bigcup_{\beta \in B_{0}(\bar{x})} \partial g_{\beta}(\bar{x})\right) .
$$

Combining (32), (37) and (38) yields that for every $x \in U$,

$$
\begin{aligned}
G(x) & =\bar{\chi}\left[\sup _{\beta \in B} g_{\beta}(x)-\sup _{\beta \in B} g_{\beta}(\bar{x})\right] \\
& \geqslant \bar{\chi} \bar{g}^{\prime}(\bar{x} ; \omega(x, \bar{x})) \\
& =\bar{\chi} \max _{\zeta \in \partial \widetilde{g}(\bar{x})}\langle\zeta, \omega(x, \bar{x})\rangle \\
& =\bar{\chi} \max _{\zeta \in \operatorname{co}\left(\bigcup_{\beta \in B_{0}(\bar{x})} \partial g_{\beta}(\bar{x})\right)}\langle\zeta, \omega(x, \bar{x})\rangle .
\end{aligned}
$$


Since $\sum_{j=1}^{\ell} \bar{\mu}_{\beta_{j}} \bar{\eta}_{j} \in \operatorname{co}\left(\bigcup_{\beta \in B_{0}(\bar{x})} \partial g_{\beta}(\bar{x})\right)$, it follows that for every $x \in U$,

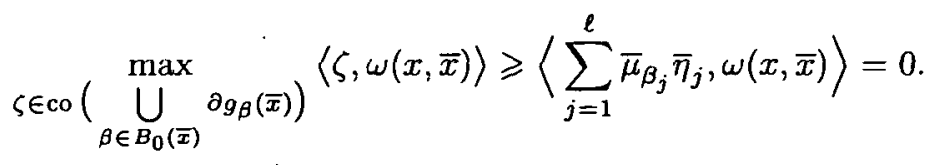

Substituting (40) into (39) yields that for every $x \in U$,

$$
G(x) \geqslant 0
$$

which conflicts with (36). This completes the proof.

Let us consider the following problem:

$$
\left(\mathrm{P}_{1}\right) \begin{cases}\text { minimise } & \sup _{\alpha \in Q} f_{\alpha}(x) \\ \text { subject to } & \sup _{\beta \in B} g_{\beta}(x) \leqslant 0\end{cases}
$$

where $Q, B, f_{\alpha}(\alpha \in Q)$ and $g_{\beta}(\beta \in B)$ are as in Problem (P).

For this problem we define the sets

$$
\begin{aligned}
& Q_{1}(\bar{x})=\left\{\alpha \in Q: f_{\alpha}(\bar{x})=\sup _{\gamma \in Q} f_{\gamma}(\bar{x})\right\}, \\
& B_{1}(\bar{x})=\left\{\beta \in B: g_{\beta}(\bar{x})=\sup _{\gamma \in B} g_{\gamma}(\bar{x})\right\} .
\end{aligned}
$$

Denote by $M$ the feasible set of $\left(\mathrm{P}_{1}\right)$ :

$$
M=\left\{x \in \mathbb{R}^{n}: \sup _{\beta \in B} g_{\beta}(x) \leqslant 0\right\} .
$$

By an argument analogous to that used for the proof of Theorem 6.1 we obtain the following

TheOREM 6.2. Let $\bar{x}$ be a local minimiser of Problem $\left(P_{1}\right)$. Assume that the functions $f_{\alpha}(\alpha \in Q)$ and $g_{\beta}(\beta \in B)$ are Lipschitzian on the same open set $U$ containing $\bar{x}$ with the same Lipschitz constants $K_{1}$ and $K_{2}$, respectively. Suppose, furthermore, that the mappings $\alpha \mapsto f_{\alpha}(\bar{x})$ and $\beta \mapsto g_{\beta}(\bar{x})$ are continuous, and the set-valued mappings $(\alpha, x) \mapsto \partial f_{\alpha}(x)$ and $(\beta, x) \mapsto \partial g_{\beta}(x)$ are upper semicontinuous at $(\alpha, \bar{x})$ $\left(\forall \alpha \in Q_{1}(\bar{x})\right)$ and $(\beta, \bar{x})\left(\forall \beta \in B_{1}(\bar{x})\right)$, respectively. Then, there exist $\bar{\sigma} \geqslant 0, \bar{\chi} \geqslant 0$, 
$\alpha_{i} \in Q_{1}(\bar{x}), \bar{\lambda}_{\alpha_{i}} \geqslant 0, i=1, \ldots, m$, with $\sum_{i=1}^{m} \bar{\lambda}_{\alpha_{i}}=1$, and $\beta_{j} \in B_{1}(\bar{x}), \bar{\mu}_{\beta_{j}} \geqslant 0$, $j=1, \ldots, \ell$, with $\sum_{j=1}^{\ell} \bar{\mu}_{\beta_{j}}=1, \bar{\sigma}$ and $\bar{\chi}$ are not both equal to zero, such that

$$
\begin{gathered}
0 \in \bar{\sigma} \sum_{i=1}^{m} \bar{\lambda}_{\alpha_{i}} \partial f_{\alpha_{i}}(\bar{x})+\bar{\chi} \sum_{j=1}^{\ell} \bar{\mu}_{\beta_{j}} \partial g_{\beta_{j}}(\bar{x}), \\
\bar{\chi} \sup _{\beta \in B_{1}(\bar{x})} g_{\beta}(\bar{x})=0 .
\end{gathered}
$$

Moreover, assume the following conditions hold

(i) There exists $\widehat{x} \in U$ such that $\sup _{\beta} g_{\beta}(\widehat{x})<0$;

(ii) For all $\beta \in B_{1}(\bar{x})$, the function $g_{\beta}$ is regular at $\bar{x}$ and invex on $U$ at $\bar{x}$ with respect to the same scale function $\omega$.

Then $\bar{\sigma}>0$, and it can be taken $\bar{\sigma}=1$.

Now we shall deal with a sufficient condition for optimality.

Theorem 6.3. Let $\bar{x}$ be a feasible point of Problem $\left(P_{1}\right)$. Assume that the functions $f_{\alpha}(\alpha \in Q)$ and $g_{\beta}(\beta \in B)$ are Lipschitzian on the same open set $U$ containing $\bar{x}$ with the same Lipschitz constants $K_{1}$ and $K_{2}$, respectively; the mappings $\alpha . \mapsto f_{\alpha}(\bar{x})$ and $\beta \mapsto g_{\beta}(\bar{x})$ are continuous; the set-valued mappings $(\alpha, x) \mapsto \partial f_{\alpha}(x)$ and $(\beta, x) \mapsto \partial g_{\beta}(x)$ are upper semicontinuous at $(\alpha, \bar{x})\left(\forall \alpha \in Q_{1}(\bar{x})\right)$ and $(\beta, \bar{x})$ $\left(\forall \beta \in B_{1}(\bar{x})\right)$, respectively. Suppose, in addition, that the following conditions are satisfied.

(a) The functions $f_{\alpha}\left(\alpha \in Q_{1}(\bar{x})\right)$ and $g_{\beta}\left(\beta \in Q_{2}(\bar{x})\right)$ are regular at $\bar{x}$, and invex on $U$ at $\overline{\boldsymbol{x}}$ with respect to the same scale function $\omega$;

(b) There are $\bar{\chi} \geqslant 0, \alpha_{i} \in Q_{1}(\bar{x}), \bar{\lambda}_{\alpha_{i}} \geqslant 0, i=1, \ldots, m$, with $\sum_{i=1}^{m} \bar{\lambda}_{\alpha_{i}}=1$, and $\beta_{j} \in B_{1}(\bar{x}), \bar{\mu}_{\beta_{j}} \geqslant 0, j=1, \ldots, \ell$, with $\sum_{j=1}^{\ell} \bar{\mu}_{\beta_{j}}=1$ such that

$$
\begin{gathered}
0 \in \sum_{i=1}^{m} \bar{\lambda}_{\alpha_{i}} \partial f_{\alpha_{i}}(\bar{x})+\bar{\chi} \sum_{j=1}^{\ell} \bar{\mu}_{\beta_{j}} \partial g_{\beta_{j}}(\bar{x}) \\
\bar{\chi} \sup _{\beta \in B_{1}(\bar{x})} g_{\beta}(\bar{x})=0 .
\end{gathered}
$$

Then $\bar{x}$ is a local minimiser for $\left(\mathrm{P}_{1}\right)$.

Proof: Let $\hat{f}(x):=\sup _{\alpha \in Q} f_{\alpha}(x)$ and $\hat{g}(x):=\sup _{\beta \in B} g_{\beta}(x)$. Applying Theorem 4.1 we claim that $\hat{f}$ and $\hat{g}$ are regular at $\bar{x}$. In view of Theorem 5.2 , it follows that $\hat{f}$ and $\hat{g}$ are invex on $U$ at $\bar{x}$ with respect to the same scale function $\omega$. 
It follows from (41) that there exist $\bar{\chi} \geqslant 0, \alpha_{i} \in Q_{1}(\bar{x}), \bar{\lambda}_{\alpha_{i}} \geqslant 0, \bar{\xi} \in \partial f_{\alpha_{i}}(\bar{x})$, $i=1, \ldots, m, \sum_{i=1}^{m} \bar{\lambda}_{\alpha_{i}}=1$, and $\beta_{j} \in B_{1}(\bar{x}), \bar{\mu}_{\beta_{j}} \geqslant 0, \bar{\eta}_{j} \in \partial g_{\beta_{j}}(\bar{x}), j=1, \ldots, \ell$, $\sum_{j=1}^{\ell} \bar{\mu}_{\beta_{j}}=1$ such that

$$
\sum_{i=1}^{m} \bar{\lambda}_{\alpha_{i}} \bar{\xi}_{i}+\bar{\chi} \sum_{j=1}^{\ell} \bar{\mu}_{\beta_{j}} \bar{\eta}_{j}=0
$$

By (42), it follows that

$$
\bar{\chi} \sup _{\beta \in B} g_{\beta}(\bar{x})=0
$$

Hence, for $x \in M \cap U$,

$$
\begin{aligned}
\sup _{\alpha \in Q} f_{\alpha}(x)-\sup _{\alpha \in Q} f_{\alpha}(\bar{x}) & \geqslant\left[\sup _{\alpha \in Q} f_{\alpha}(x)-\sup _{\alpha \in Q} f_{\alpha}(\bar{x})\right]+\bar{\chi}\left[\sup _{\beta \in B} g_{\beta}(x)-\sup _{\beta \in B} g_{\beta}(\bar{x})\right] \\
& \geqslant\langle\xi, \omega(x, \bar{x})\rangle+\bar{\chi}\langle\eta, \omega(x, \bar{x})\rangle(\forall \xi \in \partial \widehat{f}(\bar{x}), \forall \eta \in \partial \widehat{g}(\bar{x})) .
\end{aligned}
$$

Moreover, taking account of Theorem 4.1, we contend that

$$
\begin{aligned}
& \partial \widehat{f}(\bar{x})=\operatorname{co}\left(\bigcup_{\alpha \in Q_{1}(\bar{x})} \partial f_{\alpha}(\bar{x})\right), \\
& \partial \hat{g}(\bar{x})=\operatorname{co}\left(\bigcup_{\beta \in B_{1}(\bar{x})} \partial g_{\beta}(\bar{x})\right) .
\end{aligned}
$$

Substituting (43), (45) and (46) into (44) yields that

$$
\begin{aligned}
\sup _{\alpha \in Q} f_{\alpha}(x)-\sup _{\alpha \in Q} f_{\alpha}(\bar{x}) & \geqslant\left\langle\sum_{i=1}^{m} \bar{\lambda}_{\alpha_{i}} \bar{\xi}_{i}, \omega(x, \bar{x})\right\rangle+\bar{\chi}\left\langle\sum_{j=1}^{\ell} \bar{\mu}_{\beta_{j}} \bar{\eta}_{j}, \omega(x, \bar{x})\right\rangle \\
& =\left\langle\sum_{i=1}^{m} \bar{\lambda}_{\alpha_{i}} \bar{\xi}_{i}+\bar{\chi} \sum_{j=1}^{\ell} \bar{\mu}_{\beta_{j}} \bar{\eta}_{j}, \omega(x . \bar{x})\right\rangle \\
& =0 \quad(\forall x \in M \cap U),
\end{aligned}
$$

as

$$
\sum_{i=1}^{m} \bar{\lambda}_{\alpha_{i}} \bar{\xi}_{i} \in \operatorname{co}\left(\bigcup_{\alpha \in Q_{1}(\bar{x})} \partial f_{\alpha}(\bar{x})\right), \quad \sum_{j=1}^{\ell} \bar{\mu}_{\beta_{j}} \bar{\eta}_{j} \in \operatorname{co}\left(\bigcup_{\beta \in B_{1}(\bar{x})} \partial g_{\beta}(\bar{x})\right)
$$

Thus $\bar{x}$ is a local minimiser for $\left(P_{1}\right)$. 


\section{REFERENCES}

[1] A. Ben-Israel and B. Mond, 'What is invexity?', J. Austral. Math. Soc. Ser. B 28 (1986), 1-9.

[2] C. Berge, Espaces topologiques, fonctions multivoques, (Deuxième édition) (Dunod, Paris, 1966).

[3] F.H. Clarke, Optimization and nonsmooth analysis, Canadian Math. Soc. Series of Monographs and Advanced Texts (J. Wiley \& Sons, New York, 1983).

[4] B.D. Craven, 'Invex function and constrained local minima', Bull. Austral. Math. Soc. 24 (1981), 357-366.

[5] B.D. Craven and B.M. Glover, 'Invex functions and duality', J. Austral. Math. Soc. Ser. A 39 (1985), 1-20.

[6] B.D. Craven and D.V. Luu, 'Constrained minimax for a vector-valued function', Optimization 31 (1994), 199-208.

[7] B.D. Craven, D.V. Luu and B.M. Glover, 'Strengthned invex and perturbations', Math. Methods Oper. Res. 43 (1996), 319-336.

[8] M.A. Hanson, 'On sufficiency of the Kuhn-Tucker conditions', J. Math. Anal. Appl. 80 (1981), 545-550.

[9] A.D. Ioffe and V.M. Tikhomirov, Theory of extremal problems, (Russian) Nonlinear Analysis and its Applications (Izdat. Nauka, Moscow, 1974).

[10] V. Jeyakumar, 'Strong and weak invexity in mathematical programming', Methods Oper. Res. 55 (1985), 109-125.

[11] D.V. Luu and P.T. Kien, 'Sufficient optimality conditions under invexity hypotheses', Vietnam J. Math. 28 (2000), 227-236.

[12] D.V. Luu and N.X. Ha, 'An invariant property of invex function and applications', Acta Math. Vietnam. 25 (2000), 181-193.

[13] D.V. Luu and W. Oettli, 'Higher-order optimality condition for a minimax', Bull. Austral. Math. Soc. 54 (1996), 509-516.

[14] T.W. Reiland, 'Generalized invexity for nonsmooth vector-valued mapping', Numer. Funct. Anal. Optim. 10 (1989), 1191-1202.

[15] T.W. Reiland, 'Nonsmooth invexity', Bull. Austral. Math. Soc. 42 (1990), 437-446.

Institute of Cryptographic Technology

Hanoi

Vietnam
P.O. Box 631

Bo Ho

$10000 \mathrm{Hanoi}$

Vietnam 